\title{
Clinical efficacy of S-1 combined with cisplatin for advanced gastric cancer
}

\author{
Hideo Baba, Manabu Yamamoto, Kazuya Endo, Yasuharu Ikeda, Yasushi Toh, Shunji Kohnoe, \\ and TAKESHI OKAMURA \\ Department of Gastroenterologic Surgery, National Kyushu Cancer Center, 3-1-1 Notame, Minami-ku, Fukuoka 811-1395, Japan
}

\begin{abstract}
Several chemotherapy regimens used against advanced gastric cancer have been studied extensively over the decades in an attempt to further improve the prognosis of patients. To date, no standard chemotherapeutic regimens have been established worldwide. S-1 (TS-1 ${ }^{\circledR}$ ), a combination of ftorafur and two modulators, gimestat (CDHP) and oxonic acid, in a molar ratio of $1: 0.4: 1$, has been widely used in Japan for the treatment of advanced gastric cancer, and much attention has been paid to attempts to increase its antitumor effect by combining it with other chemotherapeutic drugs. We treated 12 patients with advanced gastric cancer with $80 \mathrm{mg} / \mathrm{m}^{2}$ of $S-1$ for 21 days and $60 \mathrm{mg} / \mathrm{m}^{2}$ of cisplatin (CDDP) on day 8 every 5 weeks. The treatment was continued until disease progression, unacceptable toxicity, or the patient's refusal. Eight out of 12 evaluable patients achieved a partial response (PR), with a response rate of $66.7 \%$. The incidence of grade 3 or 4 adverse effects, including myelosuppression and gastrointestinal toxicities, was $16.6 \%$. None of the patients treated with this regimen died of adverse effects and none required hospitalization for the toxicity. We conclude that the combination of S-1 and CDDP seems to have a high therapeutic index, enhancing the antitumor effect of S-1 while maintaining modest adverse effects, thus suggesting the possible use of this combination based at the outpatient clinic (apart from a short stay in hospital during the infusion of CDDP with hydration). Further study with a large number of patients may be needed to confirm the combination of S-1 and CDDP to be an appropriate first-line chemotherapy for gastric cancer.
\end{abstract}

Key words Chemotherapy $\cdot$ Gastric cancer $\cdot$ S- $1 \cdot$ CDDP

\section{Introduction}

More than 40 years after its development, fluorouracil (5-FU) remains the chemotherapeutic mainstay for the

Offprint requests to: $\mathrm{H}$. Baba

Received: November 8, 2002 / Accepted: January 28, 2003 management of patients with gastrointestinal (GI) cancer [1]. Pharmacokinetic studies have demonstrated that about $85 \%$ of clinically administered 5 -FU is inactivated and eliminated through the catabolic pathway in the liver [2]. The primary and rate-limiting enzyme related to the catabolism of 5-FU is dihydropyrimidine dehydrogenase (DPD), which metabolizes 5-FU into $\alpha$ fluoro- $\beta$-alanine [3]. A number of clinical pharmacological studies with fluoropyrimidine drugs have shown the importance of DPD to the pharmacokinetics of 5$\mathrm{FU}$, its clinical toxicity, and its tumor resistance.

Various pharmacologic strategies have been undertaken to enhance the antitumor activity of 5-FU. One such approach is to use an inhibitor of DPD. S-1 is a newly developed oral fluoropyrimidine that is a combination of the 5-FU prodrug ftorafur and two modulators, gimestat (CDHP) and oxonic acid, in a molar ratio of $1: 0.4: 1$, as reported before [4-7]. Ftorafur is converted to 5-FU through hepatic P-450s and through cytosolic enzymes [8]. CDHP is a competitive, reversible DPD inhibitor that prolongs the half-life of 5-FU. Oxonic acid is a pyrimidine phosphoribosyl transferase inhibitor that can reduce 5-FU-related GI toxicity by preventing the phosphorylation of 5-FU in the digestive tract.

In a phase I study, S-1 was administered orally for 28 days, with 2 weeks' rest. The dose-limiting factor was myelosuppression [9]. Other adverse reactions included anorexia, nausea/vomiting, and diarrhea. The pharmacokinetic profile of S-1 revealed that twice-daily ingestion could maintain the therapeutic 5-FU level without increasing Cmax in the blood.

A late phase II study of S-1 for advanced gastric cancer revealed a response rate of $49 \%(25 / 51)$ including 1 patient with a complete response (CR) and 24 patients with a partial response (PR), while the incidence of grade 3 and 4 adverse effects was less than $10 \%$ [10]. As there have been no single anticancer agents with such high antitumor effects without severe toxici- 
ties, and, moreover, as S-1 can be administered orally in an outpatient clinic, S-1 has been widely used for the treatment of advanced gastric cancer as a first-line chemotherapy in Japan [11,12].

Several efforts have been conducted to enhance antitumor effects by combining oral fluoropyrimidines with cisplatin (CDDP) or other cytotoxic agents. Ohtsu et al. [13] conducted a preliminary phase I/II study of S-1 plus CDDP for patients with advanced gastric cancer. A standard dose of $\mathrm{S}-1$ for 3 weeks and $70 \mathrm{mg} / \mathrm{m}^{2}$ of CDDP on day 8 were determined to be the maximum tolerated dose (MTD), and the recommended dose of CDDP was $60 \mathrm{mg} / \mathrm{m}^{2}$. The overall response rate was $76 \%$. Toxicity induced by S-1 combined with CDDP was slightly increased compared with that induced by $\mathrm{S}-1$ alone, but the regimen could be given safely in the outpatients clinic.

We conducted the present phase II study, using $80 \mathrm{mg} / \mathrm{m}^{2}$ of S-1 from day 1 through day 21 , with $60 \mathrm{mg} /$ $\mathrm{m}^{2}$ of CDDP on day 8 , to evaluate the antitumor effect and toxicity profile of this combination for advanced gastric cancer.

\section{Patients and methods}

\section{Eligibility}

Patients with histologically proven metastatic or recurrent gastric cancer were eligible for the study. Further eligibility criteria included measurable or evaluable lesions; performance status equal to or better than 2 on the Eastern Cooperative Oncology Group scale, with a life expectancy of 3 months or longer; no prior chemotherapy or radiotherapy, except for adjuvant chemotherapy; age less than 75 years; and adequate organ function (WBC, $\geqq 4000 / \mathrm{mm}^{3}$ but less than $12000 / \mathrm{mm}^{3}$; platelets $\geqq 100000 / \mathrm{mm}^{3}$; hemoglobin, $\geqq 9.0 \mathrm{~g} / \mathrm{dl}$; total bilirubin, $\leqq 1.5 \mathrm{mg} / \mathrm{dl}$; transaminases, $\leqq 100 \mathrm{U} / \mathrm{l}$; and creatinine, $\leqq$ upper limit of normal range). All patients provided their written informed consent.

\section{Chemotherapy regimens}

All patients were treated with S-1 combined with CDDP. S-1 was administered orally twice daily for 21 days. The dose of S-1 was based on the body surface area (BSA) as follows: $80 \mathrm{mg} /$ day for BSA less than $1.25 \mathrm{~m}^{2}, 100 \mathrm{mg} /$ day for BSA $1.25 \mathrm{~m}^{2}$ to less than $1.50 \mathrm{~m}^{2}$, and $120 \mathrm{mg} /$ day for BSA $1.5 \mathrm{~m}^{2}$ or more. CDDP, $60 \mathrm{mg} / \mathrm{m}^{2}$, was infused over $2 \mathrm{~h}$ on day 8 . Before and after CDDP administration, adequate hydration was given. This treatment schedule was repeated every 5 weeks.

Treatment administration was regulated by evaluation of blood cell count before the start of the treatment cycle. Treatment was administered if the granulocyte count was $3000 / \mu l$ or more and the platelet count was $100000 / \mu l$ or more.

\section{Evaluation of response}

The measurement and evaluation of lesions were conducted by the use of X-ray, gastrofiberscopy, computed tomography, and ultrasonography. Response was defined according to the following criteria [14]. A CR was defined as the complete disappearance of all measurable and assessable lesions for a minimum of 4 weeks. A partial response (PR) was defined as a $50 \%$ or more reduction in the sum of the products of the perpendicular diameters of measurable lesions for a minimum of 4 weeks. Progressive disease (PD) was defined as a $25 \%$ or more increase in the sum of the products of the perpendicular diameters of measurable lesions or the appearance of new lesions. Stable disease (no change; NC) was defined as other than CR, PR, or PD.

\section{Toxicity assessment}

Hematologic, mucosal, gastrointestinal, and skin toxicities were assessed before each course according to the National Cancer Institute Common Toxicity Criteria (NCI-CTC).

No prophylactic antiemetic therapy was recommended. When patients had nausea or vomiting, metoclopramide was administered. Clinical examination and blood determinations were repeated before each treatment course. Hematology laboratory evaluation was also performed weekly.

\section{Results}

During the period from March 2001 to October 2002, a total of 12 patients were enrolled. All patients had histologically proven metastatic gastric cancer, and were considered to be eligible. The characteristics of the patients are shown in Table 1. The mean age of the patients was 57.2 years. There were 3 patients with prior therapy ( 2 patients with surgery and 1 patient with chemotherapy). Differentiated and undifferentiated adenocarcinoma were found in 6 and 6 patients, respectively. Sites of metastasis included lymph node in 10 patients, liver in 6 patients, and peritoneum in 4 patients. The mean number of treatment courses given to patients was 4.3 (range, 2-10).

The responses of the patients treated with S-1 and CDDP are listed in Table 2. The overall response rate was $66.7 \%$, including eight PRs and four NCs, while there were no PDs. Response rates with regard to the site of lesions were $80 \%$ for lymph node metastasis, 
Table 1. Background of the patients treated with S-1 and CDDP

\begin{tabular}{lc}
\hline No of patients & 12 \\
Sex & 11 \\
$\quad$ Male & 1 \\
$\quad$ Female & $57.2(44-75)$ \\
Mean age; years (range) & 8 \\
PS & 3 \\
0 & 1 \\
1 & \\
2 & 9 \\
Prior treatment & 2 \\
$\quad$ None & 1 \\
Surgery & \\
Chemotherapy & 6 \\
Histology & 6 \\
$\quad$ Differentiated & \\
$\quad$ Undifferentiated & 10 \\
Sites of metastasis & 6 \\
$\quad$ Lymph node & 4 \\
$\quad$ Liver & \\
Peritoneum & \\
Average number of treatment courses (range) & $4.3(2-10)$ \\
\hline CDDP Cisplatin; PS, performance status
\end{tabular}

CDDP, Cisplatin; PS, performance status

Table 2. Response of patients treated with S-1 and CDDP

\begin{tabular}{lccccc}
\hline & CR & PR & NC & PD & RR $(\%)$ \\
& 0 & 8 & 4 & 0 & 66.7 \\
\hline Sites of lesions & & & & & \\
$\quad$ Primary $(n=10)$ & 1 & 4 & 5 & 0 & 50.0 \\
Liver $(n=6)$ & 0 & 3 & 3 & 0 & 50.0 \\
Lymph node $(n=10)$ & 2 & 6 & 2 & 0 & 80.0 \\
Peritoneum $(n=4)$ & 0 & 1 & 3 & 0 & 25.0 \\
Local $(n=1)$ & 0 & 0 & 1 & 0 & 0 \\
\hline
\end{tabular}

$\overline{\mathrm{CR}}$, Complete response; $\mathrm{PR}$, partial response; $\mathrm{NC}$, no change; $\mathrm{PD}$, progressive disease; $R R$, response rate

$50 \%$ for the liver metastasis, $50 \%$ for the primary lesion, and $25 \%$ for peritoneal seeding. There were three CRs (one in the primary lesion, and two in lymph node metastases).

Hematologic and nonhematologic adverse effects are listed in Table 3. Hematologic toxicities were often seen, but most patients demonstrated mild to moderate toxicity, of grade 1 or 2 . Two patients showed grade 3 anemia. GI toxicities, such as appetite loss, nausea, vomiting, and diarrhea were also seen; however, there were no patients with grade 3 GI toxicities.

The clinical outcome of the patients is summarized in Table 4 . Three patients were treated by surgery after the treatment with S-1 and CDDP (one patient with subtotal esophagectomy, one patient with total gastrectomy, and one patient with gastrojejunostomy). Of the12 patients four patients died of the disease and the other eight patients were still alive at the time of writing. There was no toxicity-related death.
Table 3. Toxicities in patients treated with $\mathrm{S}-1$ and CDDP

\begin{tabular}{lccccc}
\hline & \multicolumn{5}{c}{ NCI-CTC Grade } \\
\cline { 2 - 5 } & 1 & 2 & 3 & 4 & G3 or more (\%) \\
\hline Leukopenia & 3 & 5 & 0 & 0 & 0 \\
Neutropenia & 3 & 4 & 0 & 0 & 0 \\
Anemia & 1 & 7 & 2 & 0 & 16.6 \\
Thrombocytopenia & 3 & 1 & 0 & 0 & 0 \\
Appetite loss & 6 & 2 & 0 & 0 & 0 \\
Nausea & 1 & 1 & 0 & 0 & 0 \\
Vomiting & 1 & 1 & 0 & 0 & 0 \\
Diarrhea & 0 & 0 & 0 & 0 & 0 \\
Fatigue & 3 & 0 & 0 & 0 & 0 \\
Fever & 0 & 0 & 0 & 0 & 0 \\
Dermatitis & 2 & 0 & 0 & 0 & 0 \\
NCI-CTC, National cancer Institute Common Toxicity Criteria; G, \\
grade
\end{tabular}

\section{Discussion}

5-FU is one of the most commonly prescribed anticancer agents for gastrointestinal malignancy. Among several methods of administration of 5-FU, continuous i.v. infusion is superior to i.v. bolus with regard to both antitumor effects and adverse effects [15]. S-1 has been developed to increase the antitumor effect (by inhibiting DPD activity with gimestat), and also to decrease the adverse effects (by protecting the gastrointestinal tract with otastat potassium), thus resulting in a pharmacokinetic profile similar to that of continuous venous infusion of 5-FU, but with less toxicity and improved patient quality of life [4-7]. Because it is an oral preparation of fluoropyrimidine, S-1 has several advantages over the continuous venous infusion of 5-FU; the inconvenience and morbidity associated with the indwelling catheters and infusion pumps that are used with the latter treatment are avoided. Thus, $\mathrm{S}-1$ is now attracting considerable attention from oncologists [7], and it has been used for the treatment of advanced gastric cancer throughout Japan as a first-line chemotherapy.

The adverse effects of S-1 in the clinical phase I/II studies showed an incidence of less than $10 \%$ of grade $3 /$ 4 toxicities [9-12], and it therefore seems that the antitumor tumor effect of S-1 can be further enhanced by combining it with other antitumor drugs. Ohtsu et al. [13] reported a significantly high response rate of S-1 in combination with CDDP in their phase I/II study. They recommended $80 \mathrm{mg} / \mathrm{m}^{2}$ of $\mathrm{S}-1$ for 21 consecutive days, with $60 \mathrm{mg} / \mathrm{m}^{2}$ of CDDP on day 8 as an appropriate dose of this combination.

We showed, in this study, a response rate of $66.7 \%$, with minimal adverse effects, with combination chemotherapy of S-1 with CDDP. Although the response rates in our study were not as high as in the previous report, this combination of S-1 and CDDP was confirmed to 
Table 4. Outcomes of patients treated with S-1 and CDDP

\begin{tabular}{|c|c|c|c|c|c|c|c|c|}
\hline $\begin{array}{l}\text { Case } \\
\text { no. }\end{array}$ & Sex & $\begin{array}{c}\text { Age } \\
\text { (years) }\end{array}$ & PS & $\begin{array}{c}\text { No. of treatment } \\
\text { cycles }\end{array}$ & Response & $\begin{array}{c}\text { Subsequent } \\
\text { treatment }\end{array}$ & $\begin{array}{c}\text { Survival } \\
\text { time (days) }\end{array}$ & Prognosis \\
\hline 1 & $\mathrm{M}$ & 51 & 0 & 10 & PR & & 558 & Alive \\
\hline 2 & M & 61 & 0 & 6 & PR & Surgery & 541 & Alive \\
\hline 3 & $\mathrm{M}$ & 57 & 0 & 3 & PR & Surgery & 243 & Alive \\
\hline 4 & $\mathrm{M}$ & 44 & 0 & 3 & PR & & 127 & Alive \\
\hline 5 & $\mathrm{M}$ & 75 & 2 & 3 & PR & & 233 & Died \\
\hline 6 & $\mathrm{M}$ & 70 & 1 & 3 & $\mathrm{NC}$ & & 168 & Died \\
\hline 7 & $\mathrm{M}$ & 64 & 0 & 3 & $\mathrm{NC}$ & & 215 & Died \\
\hline 8 & $\mathrm{M}$ & 64 & 1 & 2 & $\mathrm{NC}$ & Surgery & 184 & Alive \\
\hline 9 & $\mathrm{~F}$ & 46 & 0 & 3 & $\mathrm{NC}$ & & 609 & Alive \\
\hline 10 & $\mathrm{M}$ & 47 & 0 & 4 & PR & & 185 & Died \\
\hline 11 & $\mathrm{M}$ & 46 & 0 & 6 & PR & & 260 & Alive \\
\hline 12 & $\mathrm{M}$ & 61 & 0 & 4 & PR & & 145 & Alive \\
\hline
\end{tabular}

have a significant therapeutic index, enhancing the antitumor effects of S-1 while maintaining modest adverse effects, thus suggesting the possible use of this combination based at the outpatient clinic (except for a short stay in the hospital during the infusion of CDDP with hydration).

The combination of S-1 and CDDP seems to be suitable for neoadjuvant chemotherapy, as well as for the treatment of metastatic gastric cancer, because it has high response rates, shown within one or two courses of administration, with minimal adverse effects that may not compromise consecutive surgery. As a response to neoadjuvant chemotherapy was reported to be able to predict prognosis in patients with advanced gastric cancer [16], the establishment of an effective neoadjuvant regimen may lead to further improvements of the prognosis. In our study, three patients were surgically treated after the chemotherapy with S-1 and CDDP; however, we did not encounter increased morbidity due to the prior chemotherapy, and we found that S-1 and CDDP could be safely administered as neoadjuvant chemotherapy. The feasibility of S-1 and CDDP as neoadjuvant chemotherapy may need to be appropriately evaluated for patients with advanced gastric cancer with bulky primary tumor or lymph nodes in future.

The disadvantages of the combination of S-1 and CDDP may include the short hospital stay during CDDP administration and the associated increase in costs and adverse effcts, as well as the difficulty of selecting second-line chemotherapy.

A clinical phase III trial comparing S-1 alone with S1 and CDDP for advanced gastric cancer has now been conducted in Japan, and the efficacy, adverse effects, and prognoses of these regimens will be evaluated appropriately in the near future.

To date, no definitive standard regimen has yet been drawn from randomized clinical trials of chemotherapy for gastric cancer, because few studies have shown a significant positive impact on survival as compared with 5-FU alone. A phase III study by the Japan Clinical Oncology Group (JCOG) has been conducted to evaluate the clinical efficacy of S-1, irinotecan (CPT-11) plus CDDP, and 5-FU alone for advanced gastric cancer. As a result of this study, the clinical usefullness of S-1 may be clarified.

We conclude that the combination of S-1 and CDDP seems to have a high therapeutic index, enhancing antitumor effects while maintaining modest adverse effects. Further study with a large number of patients may be needed to confirm the combination of S-1 and CDDP or other newly developed agents to be an appropriate firstline chemotherapy for gastric cancer.

\section{References}

1. Mayer RJ. Newer cytotoxic agents for advanced colon cancer. ASCO Educational Book. 2000. p. 625-30.

2. Heggie GC, Sommadossi JP, Cross DS, Huster WJ, Diasio RB. Clinical pharmacokinetics of 5-fluorouracil and its metabolites in plasma, urine, and bile. Cancer Res 1987;47:2003-6.

3. Diasio RB. The role of dihydropyrimidine dehydrogenase (DPD) modulation in 5-FU pharmacology. Oncology 1998;12:237.

4. Shirasaka T, Nakano K, Takechi T, Satake H, Uchida J, Fujioka A, et al. Antitumor activity of $1 \mathrm{M}$ tegafur-0.4 M 5-chloro-2,4dihydroxypyridine-1 $\mathrm{M}$ potassium oxonate (S-1) against human colon carcinoma orthotopically implanted into nude rats. Cancer Res 1996;56:2602-6.

5. Shirasaka T, Shimamoto Y, Fukushima M. Inhibition by oxonic acid of gastrointestinal toxicity of 5-fluorouracil without loss of its antitumor activity in rats. Cancer Res 1993;53:4004-9.

6. Takechi T, Nakano K, Uchida J, Mita A, Toko K, Takeda S, et al. Antitumor activity and low intestinal toxicity of S-1, a new formulation of oral tegafur, in experimental tumor models in rats. Cancer Chemother Pharmacol 1997;39:205-11.

7. Hirata K, Horikoshi N, Aibe K, Okazaki M, Denno R, Sasaki K, et al. Pharmacokinetic study of S-1, a novel oral fluorouracil antitumor drug. Clin Cancer Res 1999;5:2000-5.

8. Takiuchi H, Ajani JA. Uracil-tegafur in gastric carcinoma: a comprehensive review. J Clin Oncol 1998;16:2877-85. 
9. Taguchi T, Inuyama Y, Kanamaru R, Hasegawa K, Akazawa S, Niitani H, et al. Phase I study of S-1. Jpn J Cancer Chemother 1997;24:2253-64.

10. Sakata Y, Ohtsu A, Horikoshi N, Sugimachi K, Mitachi Y, Taguchi T. Late phase II study of novel oral fluoropyrimidine anticancer drug S-1 (1 M tegafur- $0.4 \mathrm{M}$ gimestat-1 M otastat potassium) in advanced gastric cancer patients. Eur J Cancer 1998;34:1715-20.

11. Sugimachi K, Maehara Y, Horikoshi N, Shimada Y, Sakata Y, Mitachi Y, et al. An early phase II study of oral S-1, a newly developed 5-fluorouracil derivative for advanced and recurrent gastrointestinal cancers. Oncology 1999;57:202-10.

12. Koizumi W, Kurihara M, Nakano S, Hasegawa K. The S-1 Gastrointestinal Cancer Study Group. Phase II study of S-1, a novel oral derivative of 5-fluorouracil, in advanced gastric cancer. Oncology 2000;58:191-7.
13. Ohtsu A, Boku N, Nagashima F, Koizumi W, Tanabe S, Saigenji $\mathrm{K}$, et al. A phase I/II study of S-1 plus cisplatin (CDDP) in patients with advanced gastric cancer (abstract). Proc ASCO 2001;20:165.

14. Japan Society for Cancer Therapy. Criteria for the evaluation of the clinical effects of solid cancer chemotherapy. J Jpn Soc Cancer Ther 1993;28:101-30.

15. The Meta-Analysis Group in Cancer. Efficacy of intravenous continuous infusion of fluorouracil compared with bolus administration in advanced colorectal cancer. J Clin Oncol 1998;16:3018.

16. Lowy AM, Mansfield PF, Leach SD, Pazdur R, Dumas P, Ajani JA. Response to neoadjuvant chemotherapy best predicts survival after curative resection of gastric cancer. Ann Surg 1999;229: 303-8. 